\title{
Zur Baugeschichte des Vaticans.
}

\author{
Von \\ M. Tangl.
}

Früh schon hatten sich an die alte Petersbasilica Zubauten, Klöster und Hospize gereiht. Die erste Erwähnung des Vaticans als Residenz des Papstes erhalten wir aber aus der Zeit des Schismas zwischen Laurentius und Symmachus (498-514). Für letzteren hatte sich der überwiegende Theil der Geistlichkeit entschieden, zu seinen Gunsten hatte auch der Ostgothenkönig Theodorich seinen Machtspruch gefällt; doch der Gegeupapst Jaurentius hielt den Lateran. die alte und eigentliche Papstresidenz, besetzt. So wurde denn Symmachus ron seinev Auhïngeru bei St. Peter inthronisirt, wo er ,zur Rechten und Linken eine bischöfliche Residenz errichtete " ${ }^{1}$.

Auch in der Folgezeit war und blieb der Lateran der Sitz der Päpste, soweit sie sich überhaupt in Rom aufhielten.

Leo IV. hatte nach dem Saraceneneinfall von 846 Befestigungen bei St. Peter anlegen lassen ${ }^{2}$ ), und so bot sich hier den Päpsten des 12. Jahrh. eine willkommene Zufluchtsstätte gegen das oft genug unbotmässige Rom, sofern sie überhaupt in der Stadt weilten und in den stürmischen Zeiten des Investiturstreites und der beiden Doppelwahlen voy 1130 und 1159 nicht genöthigt waren, in Frankreich Schutz zu suchen oder unstät in Tuscien und der Campagna umberzuzieben. Unter Urban II. begegnet in echten Bullen zum erstenmale die Datirung: „Romae apud beatum Petrum "(JL. 5788 ff.) und in der

1) Lib. pont. ed. Duchesne 1, 260: Tunc ab omnibus episcopis et presbiteris et diaconibus et omni clero vel plebe reintegratur sedis apostolicae beatus Sym. machus cum gloria apud beatum Petrum sedere praesul, ib. 262 item episcopia in eodem loco dextra levaque fecit, $\mathbf{g}$ l. Duchesne Notes explicatives $267 \mathrm{Nr}$. 26 : Nous avons ici le premier exemple d'un pape residant au Vatican et le premier essai de palais pontifical en cet endroit. 2) Chronica Benedicti Casis. SS. $\mathrm{rr}$. Langob. 478. 
später ausschliesslich üblichen Form: Romse apud sanctum Petrum unter Calixt II. 1120 Dec. 15 (JL 6872-74). Häufig tritt dann diese Datirung unter Eugen III. und Hadrian IV. auf ${ }^{1}$ ); - wir stehen eben in der Zeit des Versuches der Wiederbelebung der römischen Republik und Arnolds rou Brescia. Vou Eugen III. meldet uns seine Vita, dass er einen Palast bei st. Peter gebaut habe, eine Nachricht, die sich auch urkundlich belegen lässt, indem in einer 1151 Sept. 5 ausgestellten Urkunde das palatium novum des Papstes erwähnt wird ${ }^{2}$ ). Vielleicht geliugt es römischen Topographen, aus der Grenzbeschreibung die Lage des einstigen Palastes Eugen III. näher festzustellen.

Alexauder III., der bedeutendste Papst des 12. Jahrh., konnte nur zweimal auf längere Zeit seinen Sitz im Lateran aufschlagen, bei St. Peter findeu wir ihn urkundlich nur wenige Tage (JL. 11261, 13055-62, 130155). Hïufiger residirte hier Cölestin III. Grosse Bauten bei St. Peter liess dann Innocenz III. aufführen; aber die Fassung jener Stelle, welche uns davon berichtet, lässt daranf schliessen, dass es sich mehr un eine Reihe einzelner Neu- und Zubauten, als um einen grossen, nach einheitlichem Plane aufgeführten Palast handelte ${ }^{3)}$. Sprecheu schon Art und Zahl der genannten Bauten dafür, dass es sich dabei um dauerude Unterbringung eines bedeutenden Hof- und Verwaltuugspersonals handelte, so ergibt sich aus den Datirungen der Bullen, dass der Papst besonders in den .Jahren 1204-8 seinen bleibenden Sitz im Vatican gewählt hatte.

Damit hängt wohl auch zusammen, dass man nun die Register bei St. Peter, als nun vorwiegender Residenz und ständigem Sitz der Verwaltung, aufbewahrte, wodurch sie ein gütiges Geschick vor der Katastrophe verschonte, von der fast der ganze ältere Archivbestand, soweit er im Lateran und in der turris cartularia verwahrt war, um die Mitte des 13. Jahrh. betroffen wurde.

Mit Recht sieht Gregorovius ${ }^{4}$ ) den Hauptgrund zur Anlegung einer befestigten Residenz bei St. Peter in den stadtrömischen Un-

1) Vgl. Plattner, Reschreibung der Stadt Rom 2, 1. Abth. 232 Anm. Doch ist die Angabe, dass Hadrian IV. fast sein ganzes Pontificat ïber hier residirt habe, unrichtig. 2) Urkundenbeilage 1. 8) Bruchstíck aus einer gleichzeitigen Vita bei Mai, Spicilegium Romanum 6, 807 : Quia vero non tantum bonorabile sed utile censuit, nt summus pontifex apud sanctum Petrum palatium dignum haberet, fecit ibi fieri domos iatas de novo capellaniam cameram et capellam panattariam bucellariam coquinam et marescalciam domos cancellarii camerarii et eleemosynarii; aulam autem confirmari praecepit et refici logiam totumque palatium claudi muris et super portas erigi torres. Emit etiam domum intra clausuram palatii, quam ad habitationem medici deputavit. ") Geschichte der Stadt Rom im Mittelalter, 2. Aufl. 5, 613. 
ruhen und Wirren, in denen der Lateran, in dessen Nähe die Thurmpaläste des römischen Adels lagen und Hauptverkehrsstrassen wie die via Appia und Latina vorüberzogen, eine viel gefährdetere Wohnstätte war, als der Vatican, der von der übrigen Stadt durch den Tiber getrennt, durch die feste Engelsburg gedeckt und ron keiner der grossen Heerstrassen berührt war.

Der eigentliche Gründer des Vaticans als einheitlich angelegten Palastes wurde der am 25. Nov. 1277 gewählte Papst Nicolaus III. aus dem Hause der Orsini ${ }^{1}$ ). Ihm verdanken der Vatican seine Erweiterung und Neugestaltung, die vaticanischen Gärten ibre Entstehung und Anlage. Die Anhöhen nordwestlich ron St. Peter bedeckten damals meist im Besitze vou rörnischen Bürgern befindliche Weingärten. Diese liess der Papst in Laufe des Jahres 1278 durch seinen camerarius Angelus de Vezosis ankaufen und gewann so den Boden zur Anlegung der vaticanischen Gärten, die er mit Maueru und Thürmen umwallte. Bereits Gregorovius machte darauf aufmerksam, dass sich in einer Florentiner $H_{s}$. des Cencius viele Documente über diese Aukäufe findeu²).

Mit der Durchforschung des Bestaudes des Engelsburgs-Archives beschäftigt, fand ich im Armarium III. Capsa III. als Nr. 1-13 die Originaldocumente. Alle Urkunden sind auf festem, rechteckigen oder trapezförmigen Pergament von einer Hand, der des ausfertigenden Scriniarius Stephanus, in zieriicher, gewandter Minuskel gescbrieben ${ }^{3}$ ); sie sind ziemlich gut erlalten und tragen in dorso Collationirungsvermerke ${ }^{4}$ ), die wohl rou Eintragungen in eine Cencius Hs. herrühren; so Nr. 1 und 2: Collat. B. de Spello und die übrigen: Collat. per A. de Camp. et Martinum cam[ere] not[arium]. Nr. 1 überdies: in primo volumine a 118 usque ad 137 continentur hec XIII instrumenta vinearum renditionis iuxta palatium sancti Petri.

Ich gebe nur die Urkunde $\mathrm{Nr} .3$ in vollem Wortlaut wieder, weil

1) Chron. Fr. Francisci Pipini, Muratori SS. 9, 728--24: Magnopere hic (sc. Nicolaus III.,) studuit genus suum exaltare et locupletare, ut sui potentiores essent in urbe, qui habitabant regione sancti Petri. Summis sumptibus construxit palatia et pecunia collecta de?decima proventuum universarum ecclesiarum occesione passagii, quod statuerat facere Gregorius X. papa, ut ibi celebritas curiae Romanae esset in vestibulis aedium propinquorum suorum. Muratori SS. 8, 608 aus einer Hs. der Ambrosiana: Hic palatium sancti Petri multum augmen. tavit et quamplurima aedificia fieri faciens iuxta illud Parcellum inclusit et fontem ibidem fieri paravit moenibus et turribus jardinum magnum diversis arbo ribus decoratum includendo. Vgl. auch Ricordano Malespini, Istoria Fiorentina, lc. SS. 8, 1022 2) 1. c. 5, $61 \%$, Anm. 1. 3) Das besonders gross ausgefallene Perg. Nr. 2 enthält zwei Urkunden. औ) Saec. XV, 2. Hälfte. 
sie allein in der narratio den Beweggrund zu all den Grundkäuten, die Anlage des neuen Palastes, nenut, und von den andern ganz nach derselben Formel abgefassten Stücken nur lateinische Regesten mit möglichst wörtlicher Anlehnung an die Originale.

Da auf dem Pergamente Arm. III. C. VII Nr. 2 zwei Kaufverträge geschrieben wurden, erhalteu wir im ganzen 14 Urkunden, von denen sich 13 auf Neuerwerbungen und nur eine (A. III. C. VII. Nr. 7) auf einen Tauschvertrag mit dem Capitel der Kirche S. Maria in Monticellis bezieht, das für die Ueberlassung von Weingürten in monte Geretulo andere am Fusse des Monte Mario erhielt. Unter den petiae vinearum scheint, dem zwischen 8 und 29 Pfund schwankenden Kaufpreis nach zu schliessen, wohl kein bestimmtes Ackermass, sondern, entsprechend dem lavon abgeleiteten pièce, eine mehr oder minder willkürliche Grösse verstanden zu sein. Die Gesammtkosten des Ankaufes betrugen 714 l'fund "provenienses."

Die oben citirte Stelle aus der Chronik Pipins berichtet uns auch, woher die Geldmittel zum gross angelegten Unternehmen des Papstes flossen. Gregor $X$., der sich die Neuentfachung der schon verglimmenden Kreuziugsbegeisterung zur Lebensaufgabe gemacht hatte, hatte allenthalben Sammlungen angeordnet, um Streiter für die Wiedergewinuung des heiligen Landes zu rüsten und za werben. Doch der Eifer für die Kreuzzugsbewegung erkaltete bald und für immer; länger hielten sich die collectores der fijr das heil. Land bestimmten Zehenten, wie in den Jahren 1282-5 Aliron de Ricardis in unseren Gegenden in dieser. Eigenschaft thätig war $\left.{ }^{1}\right)$. Die so gesammelten Gelder dienten den Bauteu des kunstsinnigen Papstes. Gregorovius und Milanesi2) wissen auch mit grosser Bestimmtheit die Baumeister zu nennen: es seien die beiden Dominicauer Fra Sisto und Fra Ristoro gewesen, die der Papst zu dem Behufe aus Florenz berufen habe.

Nach Marchese ${ }^{9}$ ) verbält sich die Sache folgendermassen: Hauptquelle für das Leben der beiden Architekten ist ein Nekrolog ron S. Maria Novella in Florenz, wo die beiden Conversen des dazu gehörigen Dominicanerklosters und zugleich die Baumeister der Kirche waren. Nun berichtet das genannte Todtenbuch, dass der Ruhm der beiden Architekten auch nach Rom gedrungen sei und der Papst -

1) Vgl. Denifle, Archiv f. Lit. u. Kirch. Gesch. d. Mittelalt. 2, 15 Anm. S und Hauthaler, Libellus decimationis de anno 1285, Programm des Borromäums in Salzburg 1887. 2) Vasari-Ausgabe, Florenz 1878 im Commentar zur Vita des Gaddo Gaddi 1, $355-7 . \quad$ 9) , Memorie dei più insigni pittori scultori e architetti domenicani * 4. editione, Bologna 1878 cap. 3. p. 54 ff. 
der Name desselben wird nicht genannt - sie dahin berufen habe, um an seinem Palaste „die erste Gewölbeführung "1) vorzunehmen.

Nun war nach Villani der Neffe des Papstes Nicolaus III. Fr. Latino Malabranca am 8. October 1278 nach Florenz gekommen, um in dem noch immer tobenden Streit zwischen Ghibellinen und Guelfen zu vermittelu, und hatte bei der Gelegenheit an 18. October des Jahres den Grundstein zour Kirche vou S. Maria Novella gelegt. Es sei also, meint Marchese, in der That höchst wahrscheinlich, lass der berufeude Papst unser Nicolaus III. gewesen sei, dem sein Neffe die beiden Küustler, die er in Florenz kenıen gelernt hatte, zur Leitung seines Palastbaues empfohlen haben mochte; und ich nehme keinen Anstand, mich der ansprechenden Vermuthung Marcheses vollständig anzuschliessen. Danach mögen die beiden Dominicanermönche etwa 1279 nach Rom gekommen sein, wo Fra Sisto bis zu seinem Tode (März 1289) blieb, während Fra Ristoro nach kurzem Aufenthalte wieder nach Florenz zurückkehrte.

Die unmittelbaren Nachfolger Nicolaus III. baben nur wenig im Vatican residirt; zum letztenmale dauernd Bonifaz VIII. in den Wintermonaten von 1295, 96 und 97. Dann kam die avinionensische Zeit, für Rom eine Periode traurigster Verödung. Als die Päpste nach siebzigjähriger Abwesenheit wieder iu die ewige Stadt zurückkebrten, fanden sie den Lateran in tiefem Verfall, während der kurz vor dem Abzuge neugebaute Palast bei St. Peter zum bleibenden Aufenthalte einlud; er wurde die ständige Residenz der Päpste bis auf den heutigen Tag.

In Toscana war aber indessen die junge nationale Kunst zu voller Höhe emporgeblüht und lieferte den kunstsinnigen Päpsten des 15. und 16. Jahrhunderts die Männer, welche nun die grossartigen Neubauten an der Peterskirche und dem Vatican ausführten.

Theile des Palastes Nicolaus III. haben aber die für ihren Bestand gefährlichsten Umbauversuche Nicolaus $V$. überdauert und sich so bis auf unsere Tage erhalten ${ }^{2}$ ). Es sind die kleineu Höfe, die unmittelbar an den Damasushof nach Westen der Peterskirche entlang sich anreihen ${ }^{3}$ ).

1) Marchese 1. c. 75 , alcuni voltoni " das von ihı citirte Nekrolog , primas testudines." 2) Platina, de vitis pontificum (Ausgabe v. 1529) vita Nicolai III. p. 184; Aedes enim apud sanctum Petrum percommode exstruxit, quarum partem aliquam adhuc cernimus. Has postea Nicolans quintus magna impenza restituit. Cinxit etiam moenibus in urbis modum pomerium sancti Petri, quod viridarium nunc vocant. s) Auf dem Plan in den als Beilage zu Plattners etc. ,Beschreibung der Stadt Rom a augegebenen Bilderbeften I. 9 mit A bezeichnet. 
1.

1151 September 5.

Soffice ridua Mirini vendit Petro cunımico luesilice sancti Petri it suldiacono sarri palatii ius locutionis in duolus casalinis sitis in civitute Leconiuna.

Originel im vuticunischen Archiv (Arm. 1. (: X\%. . ir. 3.)

\{In nomine domini.\} Anno dominice incarnationis millesimo centesimo quinquagesimo primo, pontificatus vero domini Eugenii tertii pape anno septimo, indictione $\mathrm{XV}$., mensis Septembris die $\nabla$.

Ego quidem donna Sofa uxor quondam Miccini de Maria Pictuina hac die nullo prohibente nec contradicente aut vim faciente set propria et spontanea mea voluntate vendo et corporaliter investiens trado tibi domino Petro dei gratia canonico renerabilis basilice beati Petri apostoli et subdiacono Bacri palatii ad opus videlicet supradicti pontificis, id est totum meum ius locationis quod haben et mihi pertinet in duobus casalinis cum suis parietibus et omnibus ad ipsos pertinentibus positis in civitate Leoniana ante occlesiam sancti Vincentii et ante palatium nornm supradicti pontificis. Fines vero unius casalini sunt hii: ab uno latere est paries commune huic casalino et domui destructe filiaram mearum, al alio id est retro tenentibus heredibus Miccini mei quondam viri, ab alio tenet predicta ecclesia sancti Vincentii et a quarto est vis publica. Alterius autem casalini hii sunt fines: ab uno latere predictis heredibus Miccini tenentibus, a tribus aliis lateribus sunt rie publice. Qualiter mibi ut dictum est iure locationis pertinent, facto ab ipsa ecclesia sancti Vincentii olim Johanni Bobonis de Jaquinto, sicut in insa cartula locationis continetur et legitur et ego eos ab) heredibus ipsius Johannis emi, taliter eos tibi ut supradictum est vendo et trado pro triginta duobus solidis afforensium, quos nunc mihi coram subscriptis testibus ex camera supradicti domini pape proinde dedisti pro toto pretio. Ideoque ab hodierna die inantea licentiam et potestatem habeatis exinde faciendi quicquid robis placuerit, et nunquam a me nec ab heredibus rel successoribus meis nec etiam ab aliqua persona a nobis summissa habeatis exinde aliquam requisitionem vel contrarietatem sut litis calnmniam, set defendamus hanc venditionem vobis ab omni homine si opus et necesse fuerit. Quod si non fecerimus vel si contra hanc cartulam venire temptaverimus, conponamus vobis pro poena predictum pretium duplum et soluta poena hec eartula maneat firma, quam scribere rogavi Johannem scriniariium sanctę Romanę ecclesę in mense et indictione supraseripta $X \nabla$.

\author{
Signum † manus supradicte Sofię huius cartulę rogatricis. \\ Mons Rogeronis testis. \\ Abbas de Altebono testis. \\ Johannes Scannatus testis. \\ Guarnimentus Petri de Theofilacto testis. \\ Stefanus Octaviani de Glorioso testis.
}

(ST.) Ego Johannes (in forma Monogrammatis) scriniarius sanctę Ronanę ecclesię compleri et absolvi. 
2.

1278 Mui 9.

Andreas Barbarubea vendit Anglelo de Vezosis, domini papae cameraio medietutem duarum vinearum extra portam auream in monte Geretulo.

Original im vaticanischen Archiv (Arm. III. C. DII. Mr. 3.)

In nomine domini. Anno domini millesimo cíLXXVil, tempore domini Nicolai tertii pape, indictione sexta, mensis Maii die nona.

Andreas Barbarabea de regione sanctorum Laurentii et Damassi civis Romanus tator Symeonis, Andreotie et Agnetis nepotum suorum filiornm quondam Dauanzi germani fratris ipsius de cuius tutela plene apparet per me scriniarium infrascriptum et meo decreto et auctoritate interposits in hac parte, cam ipse tutor nec ipsi pupill infrascriptas viness retinere nequirent, propterea quod dominus papa propter nova palatia que nuper construi fecit apud palatium basilice beati Petri vineas et alias terras prope et inxts dicta palstia acquisivit et acquiri fecit per venerabilem virum dominum Angelum de Vezosis camerarium eius ad opus et utilitatem ipsins et homane ecclesie sua bona et spontanea voluntate tutorio nomine predictorum pupillorum et pro eis dedit rendidit et concessit eidem domino camerario recipienti vice et nomine domini pape eius camere pro Romana ecclesia imperpetuum medietatem duarum petiarum vinearum vites et terram cum arboribus fructiferis et infructiferis infra eam ad veram et mundam proprietatem, positas extra portam auream in Monte Geretulo, quarum dixit esse tales confines: ab uno latere est via, $a b$ aliis duobus tenet ecclesia Romana vineas quas ${ }^{a}$ ) olim fuerunt Petri Burse et dicti tutoris et ab alio est vicolus vicinalis, ad habendum tenendum possidendum et quicquid domino pape ac successoribus suis placuerit faciendum et cum omnibus accessibus et egressibus suis utilitatibus et pertinentiis. Et cessit et mandavit idem tutor nomine dictorum pupillorum et pro eis omnia iura et actiones sibi pro dictis pupillis et ipsis pupillis competentia contra omnem personam locum et universitatem, nulla ei pro dictis pupillis et ipsis pupillis penitus reservatione facta, pro pretio et nomine pretii viginti duarum librarum bonorum proveniensium senatus, quas idem tutor tatorio nomine predictorum pupillorum et pro eis confexus est et contentus se recepisse et habuisse ab ipso domino camerario et de camera domini pape sibique datum solutum et numeratum esse renuntians pro dictis pupillis exceptioni non dati non soluti et non numerati pretii et omni alii exceptioni omnino. Quod pretium depositam fuit apud presbiterum Mathiam canonicum dicte basilice ad opus et utilitatem dictorum pupillorum, quousque investiatur in re immobili pro eisdem. Quas vineas et predicta omnia idem tutol tatorio nomine predictorum pupillorum se constituit possidere $n^{\mathrm{h}}$ )[omine ipsius domin]i camerarii et camere domini pape pro Romana Ecclesia donec earum rerum possessionem acceperit corporalem, quam accipiendi deinceps auctoritate sua et retinendi licentiam dedit omnimodam. Et ad maiorem cautelam dictus tutor nomine dictorum pupillorum et pro eis constituit procuratorem et
a) so $\mathrm{A}$.
b) Auf Rasur. 
runtium specialem Petrum Buxam testem subscriptum al investiendum le predictis et aingulis et corporalem tenutsm ei tradendum et in eandem nducendum duminum camerarium supralictum vel alium quemcumque resipientem nomine camere domini pape pro ipsa Romani ecclesia imperjetuum et promisit idem tutor tutorio nomine pro eisdem pupillis ipsi lomini camerario legitime atipulanti vice et nomine domini pape eius canere et pro successoribus imperpetuum litem et controversiam in totum rel partem de predictis omnibus venditis nullo tempore non inferre nec nferenti consentire, set ea omnia tam in proprietate quam in possessione ib omni homine et universitate legitime defendere auctorizare et disbrizare et predictim venditionem et omnia ot singula que in hoc instrunento renditionis continentur perpetuo rata et firma habere observare idimplere et non contra facere vel venire per se vel alios aliqua causa rel ingenio de iure vel de facto. Et si forte predicta plus dicto pretio ralent, totum id plus idem tutor tutorio nomine pro eisdem pupillis dosavit inter vivos et remisit domino pape pro Romana ecclesia, promittens idem utor tutorio nomine pro eisdem pupillis, quol de predictis vela) aliqua parte nulli alii apparet contractus vel quasi nec in eis competit ius alicui suicumque. Quod si secus appareret et ea occasione vel alia quacumque ix vel molestia inferatur in predictis vel circa ea seu evictio aliqua evesiret, restituere dsmpna et expensas et duplum pretii persolvere nomine nteresse. Pro quibus omnibas obligavit et pignori posuit idem tator smnia bona dictorum pupillorum presentia ot futura in quibus liberam potestatem et licentiam concessit ea capere vendere et alienare usque ad ntegram satisfactionem omnium predictorum de quibus dampnis et exjensis et interesse stare roluit simplici verbo dicti domini camerarii absjue alia difficultate probationum. $\Lambda \mathrm{d}$ bec Petrus Merici de porticu sancti ?etri rogatu et precibus dicti tutoris fideiussit et promisit sepedicto domino amerario recipienti vice et nomine domini pape pro Romana ecclesia sic ie facturum et curaturum, quod piedictus tutor et predicti pupilli omnia it singula que in hoc instrumento venditionis continentur rata et firma labebunt observabunt et contra non venient aliqua ratione, et voluit, quod imnia bona sua presentia et futara essent pari inre obligata pro prelictis omnibus ipsi domino camerario pro domino papa et pro Romana :cclesis, ut bona dictorum pupillorum et in omnem causam et eventum inpliciter omninm predictorum renuntians in predictis omnibus omni seneficio iuris et facti tam canonici quam civilis et specialiter, quod non uponet primo conveniendos et exigendos predictos principales quam ipsum ideiussorem. Que omnia et singula tam dictus tutor pro dictis pupillis juam dictus fideiussor pro sese ipsis et eorum heredibus et successoribus mperpetuum repromiserunt prefato domino camerario vice et nomine do. nini pape pro Romana ecclesia imperpetuum attendere defendere et admplere et contra nullo modo vel ratione venire sub pena dicti pretii lupli, qua soluta vel non hec omnia nichilominus frma permaneant.

Actum Rome apud basilicam beati Petri in sala palatii dicti domini amerarii presente me scrinario et hiis testibus ad bec adhibitis et ropatis: scilicet domino Angelo Petri Mathei Causidico, domino Maximo iudice,

a) Zweipal geschrieben. 
domino Petro filio eius, donino magistro Jacobo de Parma clerico camere domini pape et Egidio clerico ecclesie sancte Marie in Monticellis de Aureolis.

Eodem mense die $X$. in presentia mei scriniarii et horum testium ad hec specialiter vocatorum et rogatorum domina Bonauentura uxor quondam dicti Dauanzi et mater dictorum pupillorum certiorata de omnibus iuribus suis ad predicta omnia et singula ex certa scientia consensit sponte renuntians et pacto remittens in rebus omnibus [supra] ${ }^{2}$ )dictis quantum ad prefatam venditionem attinet omnia iura ipsius competetia et competitura imperpetuum tam ypothecarum dotis et donationis propter nuptias alimentorum parafernorum et investimentorum quam omnis alterius iuris nulla ei reservatione facta, quia ei sic bene complacuit ob devotionem et reverentiam quam habet ad Romanam ecclesiam renuntians quoque Velleiano senatus consulto et beneficio nove constitutionis et omni alii auxilio. Que omnia et singula supradicta dicta domina promisit mibi scriniario tamquarn publice persone recipienti vice el nomine dicti domini pape eius camere et pro Romana ecclesia et eorum successoribus pro se eiusque heredibus et successoribus perpetuo tenere observare et contra nullo modo venire sub obligatione bonorum ipsius et pena dicti pretii pecunie dupli, qua soluta vel non bec omnia nichilominus firma permaneant. Actum ad donum predictorum pupillorum et dicti tutoris coram I'etro domini iulicis Stephani, Lauretio") Duranti et Johanne filio olim Palorzi testibus.

(S. 'T.) Ego Stephanus Johannis Guidonis civis Romanus sancte Romane ecclesie scriniarius predictis omnibus interfui subscripsi et publicavi rogatus in anno indictione mense et diebus predictis.

3.

$$
1275 \text {. April 3\%. }
$$

Puulus Riccomandi civis Romanus suo ipsius et P'tri, fratris sui, nomine vendit Angelo de Ve'zosis, domini pupae camerario tres petias vinearum extre portam auream in monte Geretulo siturum inter proprietatem he'edum Laurentii I'etri Laurentii, riam, proprietatem Barbarubece, murus urbis pro LIXX libris proceniensium senatus.

Actum Rome in sala palatii dicti domini camerarii presente me scriniario et hiis testibus ad hec adhibitis et rogatis: silicet domino Guidone, presbitero ecclesie sancte Marie in Monticellis de Aureolis Romane fraternitatis rectore, domino magistro Jacobo de Parma domini pape camere clerico, domino Calixto indice, Nicolao Johannis $\Lambda$ ngeli civibus Romanis et Thomasio clerico familiare dicti domini camerarii.

Eodom die Petrus frater Pauli Riccomandi et domina' Comtissa et Theodora, dictorum irutrum urores, r'enditioni suprascriptae consentiunt.

Actum ad domum predicturum Pauli et Petri fratrum, presentibus Petro Bussa, Nicolao Johannis Angeli et Matheo Cinthii civibus Romanis testibus ad hec vocatis et rogatis.

(S. T.) Ego Stephanus etc.

Original im vaticunizchen Archiv (Arm. HI. C. VIl. Ni. 11.)

a) Auf Rasur. b) So A. 
4.

1278 April 30.

Nicolaus Johannis Angeli de Ametiscis de regione Parrionis presente et consentiente Frederico nepote suo v'endit Angelo de Veczosis, domini papae camerario, dus petias vinearum extra portain cuream in inonte Geretulo sitarum inter viam publicam, viculum et vineas quas caineraivius a Petro et Paulo Riccomandi nuper acquisiverat pro $X L$ libris bonorum proveniensium senatus, Nicolao filio quondaı $J_{0-}$ hannis Sacunis de Ametiscis fideinssore.

Actum Rome apud hasilicam beati Petri in sala palatii dicti domini camerarii presente me scriniario et liiis testibus ad hec adhibitis et rogatis: silicet domino Guidone preshitero ecclesie sancte Marie in Monticellis de Aureolis Romane fraternitatis rectore, domino magistro Jacobo de Parma domini pape camere clericu, domino Calisto iudice, Paulo Riccomandi civibus Romanis et Thumasio clerico familiare dicti domini camerarii

Eude" dic Angeh "reor Nicohti renditioni suprascriptae consentit.

Actum ante domum dicti Nicolai venditoris presentibus Laurentio Nicolai Oddonis, Jannicone Gentilis et. L'etro Bussa testibus ad hec vocatis et rogatis.

(S. T.) Ego Stephanus etc.

Uriginal im vaticanischen Archiv (Arm. HI. C. VII. Nr. L2.)

5.

1278 Mai 1.

Juliunus et Petrus germuni fraties nuti quondam domini Laurentii Petri Laurenzii de Zerinis de regione pontis sancti Petri vendunt domino Angelo de Veczosis domini papae camerario unam vineam extra portan auream in Monte Geretulo emptan quondam a Mlatheo pesbitero rcclesie suncte Maric in Monterone rectore sitam inter proprietates Petri Bursa, Johannis Juburelli, heredum Stephani Barbarubeae et viam seu viculum vicinalem pro $\boldsymbol{X} \boldsymbol{X X I I}$ libris bonorum proveniensium senatus

Actum Rume apud basilicam beati Petri in sala palatii dicti domini camerarii presente me scriniario et hiis testibus ad hec adbibitis et rogatis: silicet Jacobo Francisci, domino Johanne Oddonis, Bruneo nepote dicti domini camerarii, Andree Barbarubee civibus Romanis, Teodino domini Raynaldi de Faria familiare dicti domini camerarii et Francisco Lombertino Florentino mercatore domini pape.

Eodem die domina Blancoflore uxor Juliani venditioni per suum maritum factae consentit.

Acturn ante domum dictorum venditorum presentibus Egidio Jacobi Morici, Pascale fabro et Petro Bussa civibus Romanis testis vocatis et rogatis.

(S. T.) Ego Stephanus etc.

Original in vaticanischen Archiv (Arm. III. C. VII. Nr. 8.)

Mittheilungen. $\mathbf{X}$. 
6.

$1278 \mathrm{Mui} 4$.

Johannes filius olim Angeli Malgerii de Pissona et Nicolaus Pincarotius de regione Parrionis v'endunt Angelo de Feczosis domini papae camerario unam petiam vineae sitam extra portam anream in monte Geretulo inter proprietates Johannis Jubarelli, Compangii Johrnnis Iucidi, Petri Bussue et viam publictim pro $X$ libris proveniensium senatus, Petro Bussa procuratore.

Actum Rome apud dictam basilicam (sc. sancti Petri) in ssla palatii dicti domini cumerarii preonte me scriniario et hiis testibas ad bec adhibitis et rogatis: scilicet domino Guidone archipresbitero ecclesie sancte Marie in Monticellis de Aureolis Romane fraternitatis rectore, domino magistro Jacobo de Parma domini papa camere clerico, domino Calixto iudice, Nicolao Johannis Angeli, Petro Bussa civibus Romanis et Thomasio familiare dicti domini camerarii.

Eodem die Jacoba uxor Johannis presente Petro de Nurgnia patre suo et Agnes uxor Nicolai venditioni per maritos suos factae consentiunt.

Actum ad domum predictorum venditorum presentibus Petro Bussa, Johannes J tabarellusa) et Contucio Ammoniti civibus Romanis testibus ad hec vocatis et rogatis.

(S. T.) Ego Stephanus etc.

Original im vaticamischen Archiv (Aim. III. C. VII. Nr. 1.)

7.

1278 Mai 4.

Andreas filius quondam Stephoni Barbarubce de regione samturum Laurentii et Damassi vendit Ang'lo de Vezosis domini papae cumerario medietatem duarum petiarum vinearum extra portam auream in monte Geretulo inter proprietutes Petri Bussae, Burtholomci et Stephani Barbarubeae, viam publicane et vicolum vicinalem pro $\boldsymbol{X} \boldsymbol{X I}$ libris proveniensium senatus, Petro Bussa procuratore.

Actum Rome apud basilicam beati Petri in sala camere palatii dicti donini camerarii presente me scriniario et hiis testibus ad hec adbibitis et rogatis: scilicet domino Calisto iudice, Matheo de Conca scriniario, Brunco nepote dicti domini camerarii civibus Romanis, domino Jacobo de Parma olerico camere domini pape et Labro cive et mercatore Lucano.

(S. T.) Ego Stephanas etc.

Original im vaticanischen Archiv (Arm. HI. C. VII. Nr. 2a.)

B) So A. 
8.

1278 Mai 4.

Johannes Jubarellus figulus civis Romanus vendit domino Angelo de Vezosis domini pupae camerario pro sancta Romana ecclesic

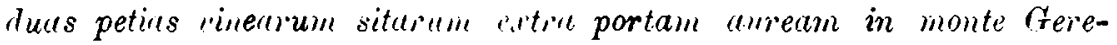
tulo inter proprietates Johannis IFalgerii et Nicolai Pincarotii et viam pro XVI libris bonorem proveniensium senatus, Ferrante Lombardo familuiri wlim domini magistri Alberti de Parma canonici basilicae sancti Petri habitatore civitatis Leonianae fideiussone.

Actum Rome apud basilicam beati Petri in sala palatii dicti domini (amerarii presente me scriniario et hiis testibus ad hec adhibitis et rogatis: silicet domino magistro Jacobo de Parma domini pape camere clerico, domino Calizto iudice, Matheus de Conca scriniarius, Brunco nepote dicti domini cumerarii et Labro cive et mercatore Lucano.

Cui renditioni eodem die etiem Muthia u.xor domini Johannis venditoris consentit.

Actum ad domum dicti venditoris coram Petro Buxa, Contucio Accomandi et presbitero Nicolao rectore ecclesie sancti Andree de Acquarizariis testibus.

(S. T.) Ego Stephanus etc.

Originul im vuticanischen Archiv (Arm. III. C. VII, Nr. 4.)

9.

1278 Mai 4.

Petrus Hugolini de Speculo de regione pontis sancti Petri civis Romanus nomine suo et Hugolini fratris vendit Angelo de Vezosis domini papae cromerario quatuor petias vinearum extra portam auream in monte Geretulo inter proprietatem Nicokii Johumis, duos viculos seu ricts et 'porprietutem Barbarabeate p'o $C$ libris proveniensium senatus

Actum Rome apud sanctum Petrum in sala palatii dicti domini camerarii presente me scriniario et hiis testibus ad hec adhibitis et rogatis: scilicet domino magistro Jabobo de Parma domini pape camere clerico, domino Calixto iudice, Labro cive et mercatore Lucano, Brunco nepote domini camerarii et Petro Bussa civibus Romanis.

Eodem mense dic V. Odolina mater et Jacoba uxor Ugolini presente patre suo Jacobo Rubeo venditioni consentiunt.

Actum ante domum predictorum fratrum presentibus Siluestro Calsolario, Romanutio Conuersano et Thoms Gracie civibus Romanis testibus ad hec vocatis et rogatis.

Eodem mense die VI. Uugulinus frater Petri venditioni consentit, Nicolao de Rusticis fideiussore. 
Actum ad balcones seu fenestras dicti palatii presente me scriniario et hiis testibus ad hec vocatis et rogatis: scilicet Bartholomeo Malialardi, Andrea Barbarubea et Cinthio Mathei Cinthii de Rusticis civibus Romanis.

(S. T.) Ego Stephanus etc.

Original im vaticanischen Archiv (Arm. III. C. VII. Nr. 5.)

10.

1278 Mai 4.

Petrus Bursa de Barbarubeis de regime sanctorum Laurentii et Damassi ciris Romanus vendit Nicoluo papae III. mediante Angelo Veczosis camerario eins unam petiam vineae sitae in monte Geretulo extra portum auream per proprictatem Andreae Barbarulei. heredum Dauanzi, heredum domini Laurentii Petri Laurentii et zicolum quendam inclusue pro $\mathbf{X X}$ libris proveniensium senatus, Nicolro Pincarutio de regione Parrionis existente fideiussore.

Actum Rome apud basilicam beati Petri in sala palatii dicti domini camerarii presente me scriniario et biis testibus ad hec adhibitis et rogatis: scilicet domino magistro Jacobo de larma domini pape camere clerico, domino Calixto iudice, Labro cive et mercatore Lucano, Brunco nepote dicti domini camerarii et Petro Bussa civibus Romanis.

Eodem die Angehe uxor Petri una cum patre suo Angelo consentit renditioni factue inter maritum sum dominique papae cameiarium.

Actum ante domum predicti venditoris coram Petro Bussa, Johanne Jubarello, Contutio Ammaniti et Andrea Barbarubea civibus Romanis testibus ad bec rocatis et rogatis.

(S. T.) Ego Stephanus etc.

Original im vaticanischen Irchiv (Arm. 1II. C. VII. Lr. 6.)

11.

1278 Mai 11.

Jacobus Rubeus de regione Parrionis civis Romremus vendit domino Angelo de Veczosis, domini pape camerario quinque petias rinearum sitarum "extr" portam auream seu castri sancti Angeli prope ecclesiam sancte Maric Madalene ad pedes Montis Mali in extremo Subereti" inter stratam Subereti, ecclesicm sanctae Mariae in Monticellis, proprietatem heredum Pauli Gottifredi et viam "que v'adit ad Suberetum" pro LXXVIII libris bonorum proveniensium senatus Johanne Occare de eaden regione fideiussore.

Actum Rome apud basilicam beati Petri in sala palatii dicti domini camerarii presente me scriniario et hiis testibus ad hec adhibitis et ro- 
gatis: scilicet domino Petro Rodulphi causidico, domino Calixto iudice, Pezcuto nepote dicti domini camerarii, Petro Buxa civibus Romanis et Gualterone clerico et familiare dicti domini camerarii.

(S. T.) Ego Stephanus etc.

"riginnl in vuticanischen Archin" (.Irm. III. C. VIII. Ii. 13.)

12.

127.8 M.ii 11.

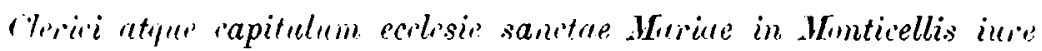

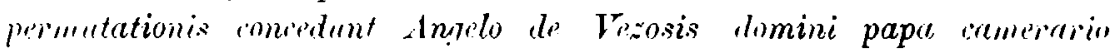

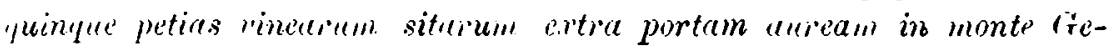

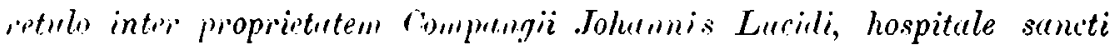

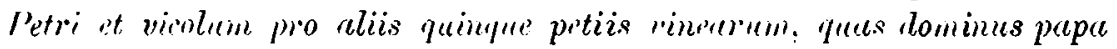
a dimino Jicoho Rubeo molo emerat.

Actum Rome apud basilicam supradictam in sala palatii dicti domincamerarii presente me scriniario et hiis testibus ad hec adhibitis et roi gatis: silicet domino Petro Rodulphi causidico, domino Calixto iudice, PetrBuxs, Gualterone familiare dicti camerarii Peczino nepote dicti domini calo merarii ot Jacobo Rubeo testibus.

(S. T.) Ego Stepbanus etc.

Original im vaticunischen Archiv (A2m. III. C. IVII. Ar. ̈.)

13.

1279 Juni 7.

Cimpragirs iartus guondain .Joluannis Lucidi de regione sancti Eustachii rendit Angelo de Veczosis domini pape camerario tres petias et dimialiam. vinearum extra portam aureerm in monte Geretulo sitarum inter viness ecclesiae Romanae emptas ab heredilus domini Laurentii Petri Lau'entii, muros urlis busilicam sancti Petri et vicolum pro $J, X$ libris bonorum proveniensium senctus.

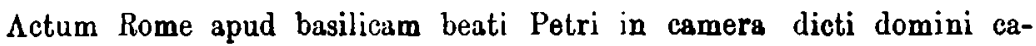
merarii presente me scriniario et hiis testibus ad hec adbibitis et rogatis: silicet Yetro Johannis Peezati, domino Angelo Peczengaro indice, Petro Bussa civibus Romanis, domino Gentile familiare et clerico dicti domini camerarii, Franeisco Lambertino de Florentia et Molario cursore.

Eodem mense die VIII Bartholomea uxor Compagii et Aldruda u.cor. Johannis filii ipsins renditioni consentiunt.

Actum ad domum dicti Compagii venditoris presentibus Johanne Montefalcho, Petro Bussa, Nicolao Barii et Bartholomeo Angeli de Terme clerico civibus Romanis testibus.

(S. T.) Ego Stephanus etc.

Original im valicanischen Archiv (Arm. III. C. VII. . Vr. 9.) 
14.

1278 Juni 9.

Magister Nicolaus scriptor domini papae et rector ecclesiae sancti Egidii extra portam auream vendit domino Angelo de Veczosis domini papae camerario $X V I I I$ petias vinearum in monte sancti Egidii seu monte Geretulo sitarum inter viam, "que vadit per ipsum montem," proprietates Romani Johannis, Johannis Stephani, Sregorii Petri Rubei et hortum post casarinum ipsius ecclesiae pro CCX libris bonorum proveniensium senatus.

Actum Rome apud basilicum beati Petri in camera Palatii domini camerarii supradicti presente me scriniario et hiis testibus ad hec adhibitis et rogatis: silicet domino Nicolia de Spoleto domini pape cappellano cantore Auersano Paulo Riccomandi, Egidio clerico ecclesie sancte Marie in Transtiberim, Laurentio Capiti, Thoma et Alexandro clericis et familiaribus dicti domini camerarii et Brunco nepote dieti domini camerarii.

(S. T.) Ego Stephanus etc.

Original im vaticanischen Archiv (Arm. III. C. VII. Nr. 10.)

15.

1278 Juni 10.

Robertus Jarobi de regione rampi Martis vendit Angelo de Veczosis: domini Nicolai papae III. camerario tres petias vinearum sitarum extra portam auream in monte Geretulo inter rlirem vineam a Romana ecclesia modo emptam, basilicam sancti Petri, muros urbis et vculum publicum pro $\boldsymbol{X X X V}$ libris bonorum proveniensium senatus, Jacobo Stephani Garzellone et Contutio Aminaniti de regione porticus sancti Petri fideiussoribus.

Actum Rome apud dictam basilican in palatio dicti domini camerarii presente me scriniario et hiis testibus ad hec adhibitis et rogatis, silicet domino magistro Alberto de Parma canonico dicte basilice, domino magistro Jacobo de Parma clerico dicte camere, domino Gentile clerico dicti domini camerarii, Petro Buxa, Angelo Petri Roberti, presbitero Somao rectore ecclesie sanoti Benedicti Sconzi, Ariano Petri Bufi et Contucio Accomandi.

(S. T.) Ego Stephanus etc.

Original im vaticanischen Archiv (Arm. III. C. DII. Nr. 2b). 